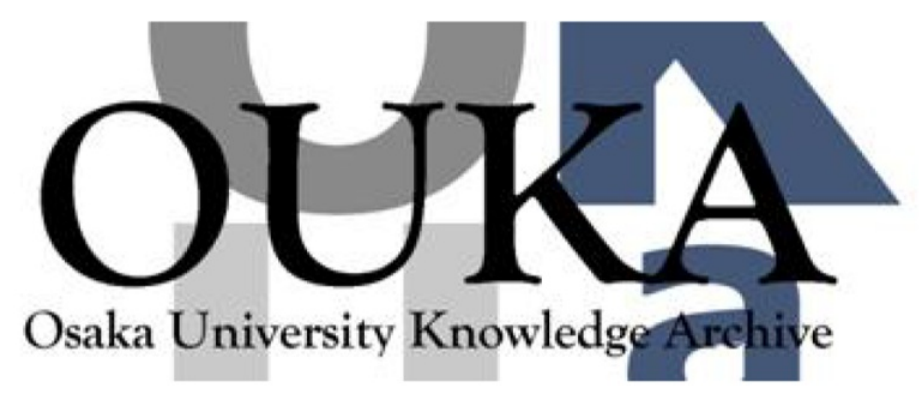

\begin{tabular}{|c|l|}
\hline Title & $\begin{array}{l}\text { Acetylation of optically transparent cellulose } \\
\text { nanopaper for high thermal and moisture } \\
\text { resistance in a flexible device substrate }\end{array}$ \\
\hline Author(s) & Yagyu, Hitomi; Ifuku, Shinsuku; Nogi, Masaya \\
\hline Citation & Flexible and Printed Electronics. 2(1) p. 014003 \\
\hline Issue Date & $2017-03-17$ \\
\hline oaire:version & AM \\
\hline URL & https://hdl. handle.net/11094/78452 \\
\hline rights & ○ 2017 IOP Publishing Ltd \\
\hline Note & \\
\hline
\end{tabular}

Osaka University Knowledge Archive : OUKA

https://ir. Library. osaka-u. ac. jp/

Osaka University 


\title{
Acetylation of optically transparent cellulose nanopaper for high thermal and moisture resistance in a flexible device substrate
}



1 The Institute of Scientific and Industrial Research, Osaka University, Mihogaoka 8-1, Ibaraki, Osaka 567-0047 Japan.

${ }^{2}$ Graduate School of Engineering, Tottori University, Tottori 680-8533, Japan

*Corresponding author

E-mail: nogi@eco.sanken.osaka-u.ac.jp.

\begin{abstract}
Optically transparent cellulose nanopaper is a promising candidate for flexible device substrates because they are lightweight and have, a smooth surface, and high dimensional stability against temperature. However, the moisture and thermal resistance should be improved, while maintaining high transparency for application. In a transparent polymer composite reinforced with cellulose nanofibers, these properties were improved by cellulose acetylation, which were fabricated by starting from acetylated pulps or rinsing acetylated nanofiber film with water. However, these acetylation procedures reduce the transparency of cellulose nanopaper because the hydrophobic nature of acetyl groups produce inhomogeneous aggregations of cellulose nanofibers, which cause light scattering. Therefore, we propose a new procedure of acetylated cellulose nanopaper by rinsing with low polarity solvents such as toluene. Obtained acetylated cellulose nanopaper shows improved moisture and thermal resistance, and maintains optical transparency. The moisture and thermal resistance increase with the degree of acetyl group substitutions. The total transmittance, haze, crystallinity, coefficient of thermal expansions, dielectric constant, and dielectric loss are constant for varying DSs. These findings promote the application of transparent cellulose nanopaper in flexible device substrates.
\end{abstract}

\section{Introduction}

Cellulose nanopaper contains only cellulose nanofibers, which were isolated from plant cell walls. Because cellulose nanofibers are densely packed, the nanopaper has a high optical transparency and smooth surface [1, 2]. The nanopaper exhibited a low coefficient of thermal expansion (CTE) similar to that of glass, and a 
higher thermal durability and dielectric constant than those of most plastics because of the high mechanical properties of cellulose nanofibers [1-4]. Moreover, the nanopaper was still lightweight and maintained a high foldability similar to conventional paper. Given these advantages, flexible electronics using cellulose nanopaper have been reported, such as transparent electrodes, organic solar cells, transistors, antenna, and memory [4-13]. Therefore, transparent cellulose nanopaper is a promising substrates for future flexible devices.

When we look at applications of transparent nanopaper in flexible devices, the hygroscopicity and thermal resistivity are big drawbacks during manufacturing and use. To overcome these problems, the properties of cellulose nanopaper have been evaluated in device applications $[2,14,15]$. When cellulose nanopaper was heated to over $150{ }^{\circ} \mathrm{C}$ in air, the optical transparency was more stable than those of common plastics such as a polyethylene terephthalate film $[2,15]$. When the cellulose nanopaper was exposed to $85^{\circ} \mathrm{C}$ and a humidity of $85 \%$, conductive lines printed on them maintained a high conductivity for at least 1000 h [14]. Furthermore, Shimizu et al., reported that the water-resistance of cellulose nanopaper was improved, while maintaining high optical transparency, by ion-exchange treatments [16, 17]. However, these studies have individually discussed the moisture or thermal durability, but not the hygroscopicity, thermal durability, and high optical transparency. When the transparent nanopaper obtain these properties, the manufacturing process and the applications in the flexible devices will be more attractive. Moreover, the device performance and reliability will improve. Therefore, the improvement of moisture and thermal resistance in nanopaper, while maintaining a high optical transparency, is one of the most important topics for future flexible devices.

Acetylation, where the hydroxyl groups of cellulose are replaced by less hydrophilic acetyl groups, is widely used for the improvement of moisture and thermal resistance of cellulose nanofiber materials [18-22]. There are two approaches to fabricate acetylated cellulose nanofiber films. First, cellulose nanofibers are mechanically nanofibrillated from acetylated microsized cellulose pulp fibers, and cellulose nanofiber films are fabricated using the acetylated nanofibers [23]. Second, a cellulose nanofiber film is obtained using native cellulose nanofibers, and then acetylated [19, 20]. These acetylated cellulose nanofiber films were opaque because they contain air cavities causing a mismatch of refractive indexes and light scattering [24, 25]. When the acetylated films were impregnated with transparent polymer, obtained composites showed a high transparency because 
the refractive index mismatches were suppressed. These transparent composites reinforced with acetylated nanofibers exhibited higher moisture and thermal resistance compared with the composites reinforced with native cellulose nanofiber $[19,20]$.

Because the cellulose nanopaper does not contain air cavities, they were highly optical transparent without the impregnation of any polymers [1, 2]. The transparency enhancement is different from that in nanofiber-polymer composites. Therefore, another acetylation process should be developed for the transparent cellulose nanopaper to improve the moisture and thermal resistance, while maintaining high optical transparency. In this study, we propose an acetylation process for transparent cellulose nanopaper and evaluate the properties depending on the degree of substitution (DS) of acetyl groups.

\section{Experimental}

\section{Cellulose pulp, nanofibrillation, and nanopaper fabrication}

Holocellulose pulps were prepared from wood chips of Japanese cedar (Cryptomeria japonica). The procedure is summarized as follows. Wood chips (40 g) were dewaxed by stirring in an acetone/water mixture (900 mL:100 mL) at room temperature overnight. The chips were delignified by heating in an acetic anhydride/hydrogen peroxide mixture $(500 \mathrm{~mL}: 500 \mathrm{~mL})$ at $90{ }^{\circ} \mathrm{C}$ for $2 \mathrm{~h}$.

The cellulose pulps were disintegrated using a water-jet nanofibrillation. In this system, a $0.5 \mathrm{wt} \%$ pulp water dispersion of $2000 \mathrm{~g}$ was homogenized using a highpressure water-jet system (Star Burst, HJP-25005 E, Sugino Machine Co., Ltd.) equipped with a ball-collision chamber. The slurry was ejected from a small nozzle with a diameter of $0.17 \mathrm{~mm}$ under a high pressure of $245 \mathrm{MPa}$. The water dispersions were passed through this nozzle up to 50 times. Cellulose nanofiber/water dispersions were condensed to 0.8 wt\% using an evaporator. The dispersions were dropped onto an acrylic plate and oven-dried at $50{ }^{\circ} \mathrm{C}$ overnight. After drying, a transparent $40-\mu \mathrm{m}$ thick nanopaper was obtained.

\section{Acetylation}

The nanopaper $(70 \times 70 \mathrm{~mm})$ was dehydrated by acetone and placed in a Petri dish containing a mixture of $40 \mathrm{~mL}$ of acetic acid, $50 \mathrm{~mL}$ of toluene, and $0.25 \mathrm{~mL}$ of $60 \%$ perchloric acid. The desired amount of acetic anhydride (3, 5, or $10 \mathrm{~mL}$ ) was added with stirring. The mixture was allowed to stand for 1 or $3.5 \mathrm{~h}$ at room temperature. After acetylation, the samples were washed thoroughly with water, methanol, ethanol, toluene, or isopropanol. Because of the addition of toluene as a 
poor solvent into the acetylation reaction system, cellulose nanofiber shapes were retained, even in the high-DS sample, although cellulose acetate fibers were dissolved in acetic acid at the surface [20].

\section{DS of acetylated nanopaper}

To determine the DS of acetylated nanopaper, a calibration curve was prepared by back titration and Fourier transform infrared (FTIR) spectroscopy of acetylated softwood dissolving sulfite pulps [18, 20,22]. The back titration was performed as follows. Acetylated cellulose pulp of $0.1 \mathrm{~g}$ was placed into $40 \mathrm{~mL}$ of $75 \%$ aqueous ethanol, and then heated at $50-60^{\circ} \mathrm{C}$ for $30 \mathrm{~min}$ to promote swelling of the material. A $40-\mathrm{mL} 0.5 \mathrm{~N} \mathrm{NaOH}$ solution, accurately measured, was added to the sample. The mixture was stirred at $50-60^{\circ} \mathrm{C}$ for $30 \mathrm{~min}$ and then stirred at room temperature for $60 \mathrm{~min}$. The excess alkali was titrated with $0.5 \mathrm{~N} \mathrm{HCl}$ using phenolphthalein as an indicator. Infrared spectra of the acetylated samples were measured with an FTIR spectrometer (Frontier TN, Perkin-Elmer Japan Co., Ltd) equipped with an ATR attachment (Universal ATR, Perkin-Elmer Japan Co., Ltd). All the spectra were obtained by an accumulation of four scans at a resolution of $4 \mathrm{~cm}^{-1}$ in the range 400 $4000 \mathrm{~cm}^{-1}$. The peak intensity at around $1730 \mathrm{~cm}^{-1}$ was recorded after these spectra were normalized by an absorption band at $1428 \mathrm{~cm}^{-1}$ derived from the cellulose main chain. From these results, a calibration curve DS $=0.0756 \mathrm{~A}-0.0409\left(\mathrm{r}^{2}=0.9921\right)$ was determined (figure 1). The DS of acetylated nanopaper was derived from the peak intensity at around $1730 \mathrm{~cm}^{-1}$ and the calibration curve.

\section{Characterizations}

Total transmittance and haze were measured using a haze meter (HZ-V3, Suga Test Instruments Co., Ltd.). Even transparent materials without any light absorption suffer reduced transparency because of light scattering. This light scattering is called haze.

The measurements of moisture content were as follows. Samples were treated for $3 \mathrm{~d}$ at $55 \%$ relative humidity and $20^{\circ} \mathrm{C}$ with a saturated aqueous solution of $\mathrm{Mg}\left(\mathrm{NO}_{3}\right)_{2}$. The sample was oven-dried at $105^{\circ} \mathrm{C}$ for $24 \mathrm{~h}$, and the moisture content was determined from the oven-dried weight.

Contact angle formed by a distilled water droplet after $20 \mathrm{~s}$ of impact on the nanopaper was measured using a contact angle analyzer (Drop Master 300, Kyowa Interface Science Co. Ltd.). Using $2-\mu \mathrm{L}$ water droplets, five sets of measurements were performed at different locations on the nanopaper and averaged.

X-Ray diffraction (XRD) patterns were recorded using a Rigaku MiniFlex600 with $\mathrm{Cu}-\mathrm{Ka}$ radiation and a scanning angle $(2 \theta)$ range of $5-40^{\circ}$ at $40 \mathrm{kV}$ voltage and 
$15 \mathrm{~mA}$ current. The crystallinity index of cellulose I was calculated from the (200) reflection $\left(2 \theta=\mathrm{ca} .22 .6^{\circ}\right)$ as previously described [26].

The CTE was measured using a thermomechanical analyzer (TMA/SS6100, SII Nanotechnology Inc.). The CTE values were determined as the mean values at 20 $150{ }^{\circ} \mathrm{C}$ in the second run.

The dielectric properties were measured using an impedance analyzer (E5071C; Agilent Technologies, Inc.) with a split post dielectric resonator (QWED) at $1.1 \mathrm{GHz}$. Prior to measurement, samples were stored in a humidity chamber (SH-641; ESPEC Co., Ltd.) at $23{ }^{\circ} \mathrm{C}$ and $50 \%$ relative humidity overnight.

The yellowness index (YI) was measured by a color meter (ZE 6000, Nippon Denshoku Industries Co., Ltd.). When the nanopaper was heated, the appearance changes from colorless transparent to yellow transparent. The discoloration is described using the differential YI $(\Delta \mathrm{YI})$, which is the color change before and after heating.

\section{Results and Discussion}

The cellulose nanopaper was fabricated by drying the cellulose nanofiber/water dispersions, which were mechanically nanofibrillated pulp fibers. When the holocellulose pulp/water dispersions were repeatedly nanofibrillated 50 times, those at $0.1 \mathrm{wt} \%$ showed a high transmittance of $88.7 \%$, and a high transparent cellulose nanopaper with $9 \%$ haze was obtained after drying the dispersion (figure $2 \mathrm{a}$ ). They were expected transmittance at $0.1 \mathrm{wt} \%$ dispersion and expected haze at $40-\mu \mathrm{m}$ thick nanopaper.

At first, we tried to fabricate acetylated transparent nanopaper from acetylated pulp fibers. When the acetylated holocellulose pulp/water dispersions were mechanically nanofibrillated 50 times, the $0.1 \mathrm{wt} \%$ dispersions were turbid and their transmittance was only $16.2 \%$. Increasing the mechanical nanofibrillation cycles disintegrated the pulp fibers into nanofibers [26]. Because the low transparent dispersion created a high haze transparent nanopaper, the nanofibrillation process was increased to 100 times. However, after 100 times nanofibrillation, acetylated cellulose nanofibers were difficult to disperse into the water homogeneously because of their hydrophobic nature. Thus, the transmittance of acetylated nanofiber/water dispersions was only $39.0 \%$, and the dried film of nanopaper was translucent with a high haze of $84 \%$ (figure $2 b$ ).

Second, we tried to fabricate the acetylated transparent cellulose nanopaper by the acetylation of transparent cellulose nanopaper. The transparent cellulose 
nanofibers from native cellulose nanofiber were densely packed by hydrogen bonding inside the nanopaper. Therefore, the nanopaper maintained high optical transparency during immersion into the reagents of acetic acid and toluene. However, when the acetylated nanopaper was immersed into distilled water to rinse the reagents, the acetylated nanopaper became translucent within 20-30 s. After drying this rinsed nanopaper, the transparency did not recover to the original haze value, which doubled from $9 \%$ to $18 \%$ (figure 2 c). The transparency loss was induced by water swelling of cellulose in the nanopaper, which created cavities and caused the light to scatter. When the acetylated nanopaper without rinsing or with an insufficient water rinsing were dried, the nanopaper became yellow and brown transparent because of the residual reagents. Thus, the reacted nanopaper were rinsed with ethanol, methanol, isopropanol, or toluene to maintain dense aggregations between cellulose nanofibers during the rinsing (figure $2 \mathrm{~d}-\mathrm{g}$ ). During the rinsing and after drying, the nanopaper showed high optical transparency because of a low polarity. Acetylated nanopaper rinsed with toluene exhibited the lowest haze of $10.5 \%$. Nanopaper from acetylated pulp fibers or acetylated nanopaper rinsed with water lost transparency. However, when the rinsing agent was changed from water to low polarity solvents, acetylated nanopaper with high transparency was successfully obtained.

The properties of acetylated cellulose were evaluated for varying DSs by adjusting the amount of acetic anhydride and reaction time [18, 20]. When a cellulose nanopaper of $70 \times 70 \mathrm{~mm}$ square and $40-\mu \mathrm{m}$ thickness was treated with acetic anhydride of $3 \mathrm{~mL}$ and other reagents for $1 \mathrm{~h}$, a DS of 0.36 was obtained. Increasing of the anhydride amount or the time increased the DS from 0.36 to $0.50,1.13$, or 1.29.

In the transparent polymer composites reinforced with cellulose nanofibers, the transparency was increased by the small refractive index gaps between polymer matrix and the cellulose nanofibers [25]. Increasing the DS of acetylation decreased the refractive index of cellulose nanofibers. For example, when the polymer with a lower refractive index than native cellulose was reinforced with acetylated cellulose nanofibers, the increasing DS showed a refractive index of nanofibers similar to that of polymer, and the composites obtained a high transparency [20]. However, increasing the DS further decreased the transparency of composites because the refractive index of acetylated nanofiber became lower than that of the matrix polymer [20]. The transparency of acetylated nanofiber composites depends on the DS of cellulose nanofibers. However, cellulose nanopaper contains only cellulose nanofibers without any polymer. Thus, the total transmittance was constant around 
$90 \%$ for all the DSs of cellulose nanofibers (figure 3a). Moreover, the hazes of acetylated nanopapers were constant around $8-10 \%$ for various DSs from 0.36 to 1.29 (figure $3 b$ ). The haze was produced by light scattering inside or at the surface of the transparent nanopaper [2]. Therefore, the acetylation procedure maintained homogeneous nanofiber aggregations and surface smoothness in the cellulose nanopaper.

Cellulose and cellulose nanofibers are highly hygroscopic materials, and the hygroscopicity can be reduced by acetylation. For example, when hardwood bleached kraft pulps were acetylated, the moisture content was linearly decreased from 5.9 $\mathrm{wt} \%$ at $\mathrm{DS}=0$ to $3.1 \mathrm{wt} \%$ at DS $=1.9$ (figure 4 ). As previously shown in polymer composites reinforced with acetylated cellulose nanofibers, low acetylation less than $\mathrm{DS}=0.56$ decreased the hygroscopicity, but excessive acetylation over $\mathrm{DS}=0.56$ increased it [20]. However, the moisture content of transparent nanopaper was linearly decreased until $\mathrm{DS}=1.29$, which was similar to that of acetylated pulp fibers. When the DS was 1.29 , the moisture absorption was about $30 \%$ lower than that of native cellulose nanopaper. The transparent nanopaper in this study was fabricated using holocellulose pulps, which contained cellulose and hemicellulose. Given the higher hygroscopicity of hemicellulose than cellulose, the transparent nanopaper exhibited larger moisture contents than hardwood bleached kraft pulps. When the hemicellulose was reduced by alkali treatment, the hygroscopicity was drastically decreased, and a high optical transparency was maintained [27].

These results show that acetylated nanopaper enhanced hydrophobicity with increasing DS. Therefore, a water droplet on the higher DS acetylated nanopaper provided a larger contact angle (figure 5). For example, the contact angle was $70^{\circ}$ on the acetylated nanopaper with DS $=1.29$. In the fabrication of flexible devices, patterns are often inkjet printed with conductive inks. After printing and sintering, the ink droplets with high contact angles became a sharp and narrow conductive pattern with a high electric conductivity [28, 29]. Therefore, acetylated nanopapers with a high DS have a high potential for future flexible device substrates because fine conductive patterns can be printed on them.

A high thermal resistance of transparent cellulose nanopaper is one of the advantages for flexible device substrate applications. As shown in a previous study, the clear transparency in a polyethylene terephthalate film was lost by heating to $150{ }^{\circ} \mathrm{C}$ in air, but the cellulose nanopaper retained a clear transparency [2]. In this study, the thermal stability of color change was evaluated by $\Delta \mathrm{YI}$, which is a number calculated from spectrophotometric data that describes the change in color of a test 
sample from clear or white to yellow. When a cellophane film was heated at $200{ }^{\circ} \mathrm{C}$ in air, its $\Delta \mathrm{YI}$ reached 15 within $10 \mathrm{~min}$. However, the transparent cellulose nanopaper without acetylation needed $20 \mathrm{~min}$ for $\Delta \mathrm{YI}=15$ (figure 6). The acetylation of cellulose improved thermal stability because of the protection of the hydroxyl groups in cellulose [22]. Therefore, the duration for reaching a $\Delta \mathrm{YI}$ of 15 was extended to $40 \mathrm{~min}$ in the acetylated cellulose nanopaper with $\mathrm{DS}=0.50$, and $60 \mathrm{~min}$ in the nanopaper with DSs $=1.13$ and 1.29. The low-temperature processes for flexible devices have been developed by printed electronics; however, a higher temperature increases the device performance. Therefore, the acetylation of transparent nanopaper will contribute to flexible device processing.

Transparent cellulose nanopaper have other advantages for flexible device substrates such as low CTE and a high dielectric constant. Given the high crystallinity of cellulose nanopaper, non-acetylated cellulose nanopaper has a low CTE of $8.0 \mathrm{ppm} / \mathrm{K}$, which is as low as glass, and a high dielectric constant of 6.9, which is as high as polyvinylidene difluoride. Because the transparent cellulose nanopaper were surface acetylated in this study, all the acetylated nanopapers maintained a high crystallinity, which was as high as the native cellulose nanopaper (figure 7a). Therefore, the acetylated cellulose nanopaper maintained CTEs of 8.0$11.1 \mathrm{ppm} / \mathrm{K}$, high dielectric constants of 5.6-6.9, dielectric losses of $0.17-0.26$ for the various DSs of 0.36-1.29 (figure $7 \mathrm{~b}-\mathrm{d}$ ). These properties allow for application as a downsized antenna for use in wearable wireless communications and a high-mobility organic thin film transistor array following a similar process to that used for an array on conventional glass [4, 8]. The material properties of transparent cellulose nanopaper, acetylated cellulose nanopaper, and other transparent polymers are presented in the supporting information (table S1). These results encourage further developments of the properties of transparent cellulose nanopaper for future flexible device applications.

\section{Conclusion}

In this study, we improve the moisture and thermal resistance of cellulose nanopaper, while maintaining optical transparency. An optically transparent cellulose nanopaper was surface acetylated, and rinsed with low polarity solvents. The acetylated cellulose nanopaper decreased moisture absorption under $55 \% \mathrm{RH}$ at $20{ }^{\circ} \mathrm{C}$, improved heat resistance against $200^{\circ} \mathrm{C}$ in air, and maintained high optical transparency, low haze, high crystallinity, low coefficient of thermal expansion, and a high dielectric constant. A higher degree of substitution in acetylated cellulose 
nanopaper improved moisture and thermal resistance. The acetylation of transparent nanopaper will contribute to flexible device processing.

\section{Acknowledgments}

This work was financially supported by Grants-in-Aid for Scientific Research (grant no. 26220908) from the Japan Society for the Promotion of Science (JSPS).

\section{References}

[1] Nogi M, Iwamoto S, Nakagaito A N and Yano H 2009 Optically transparent nanofiber paper Adv. Mater. 21 1595-8

[2] Nogi M, Kim C, Sugahara T, Inui T, Takahashi T and Suganuma K 2013 High thermal stability of optical transparency in cellulose nanofiber paper Appl. Phys. Lett. 102181911

[3] Saito T, Kuramae R, Wohlert J, Berglund L A and Isogai A 2013 An ultrastrong nanofibrillar biomaterial: the strength of single cellulose nanofibrils revealed via sonication-induced fragmentation Biomacromolecules 14 248-53

[4] Inui T, Koga H, Nogi M, Komoda N and Suganuma K 2015 A miniaturized flexible antenna printed on a high dielectric constant nanopaper composite Adv. Mater. 271112 6

[5] Hsieh M C, Kim C, Nogi M and Suganuma K 2013 Electrically conductive lines on cellulose nanopaper for flexible electrical devices Nanoscale 5 9289-95.

[6] Zhu H, Fang Z, Preston C, Lia Y and Hu L 2014 Transparent paper: fabrications, properties, and device applications Energy Environ. Sci. 7 269-87.

[7] Fang Z, Zhu H, Preston C and Hu L 2014 Development, application and commercialization of transparent paper Transl. Mater. Res. 1015004.

[8] Fujisaki Y, Koga H, Nakajima Y, Nakata M, Tsuji H, Yamamoto T, Kurita T, Nogi M and Shimidzu N 2014 Transparent nanopaper-based flexible organic thin-film transistor array Adv. Funct. Mater. 24 1657-63.

[9] Koga H, Nogi M, Komoda N, Nge T T, Sugahara T and Suganuma K 2014 Uniformly connected conductive networks on cellulose nanofiber paper for transparent paper electronics NPG Asia Mater. 6 e93.

[10] Nagashima K et al. 2014 Cellulose nanofiber paper as an ultra flexible nonvolatile memory Sci. Rep. 45532.

[11] Nogi M, Karakawa M, Komoda N, Yagyu H and Nge T T 2015 Transparent conductive nanofiber paper for foldable solar cells Sci. Rep. 517254. 
[12] Yei Hwan Jung et al. 2015 High-performance green flexible electronics based on biodegradable cellulose nanofibril paper Nat. Commun. 67170.

[13] Kang W, Yan C, Foo C Y and Lee P S 2015 Foldable electrochromics enabled by nanopaper transfer method Adv. Funct. Mater. 25 4203-10.

[14] Nge T T, Nogi M and Suganuma K 2013 Electrical functionality of inkjet-printed silver nanoparticle conductive tracks on nanostructured paper compared with those on plastic substrates J. Mater. Chem. C 1 5235-43

[15] Yagyu H, Saito T, Isogai A, Koga H and Nogi M 2015 Chemical modification of cellulose nanofibers for the production of highly thermal resistant and optically transparent nanopaper for paper devices ACS Appl. Mater. Interfaces 7 22012-7.

[16] Shimizu M, Saito T and Isogai A 2014 Hydrophobic, Ductile, and Transparent Nanocellulose Films with Quaternary Alkylammonium Carboxylates on Nanofibril Surfaces Biomacromolecules 15 4320-4325

[17] Shimizu M, Saito T and Isogai A 2016 Water-resistant and high oxygen-barrier nanocellulose films with interfibrillar cross-linkages formed through multivalent metal ions J.Membr. Sci. $\mathbf{5 0 0} 1-7$

[18] Kim D-Y, Nishiyama Y and Kuga S 2002 Surface acetylation of bacterial cellulose Cellulose 9 361-7

[19] Nogi M, Abe K, Handa K, Nakatsubo F, Ifuku S and Yano H 2006 Property enhancement of optically transparent bionanofiber composites by acetylation Appl. Phys. Lett. 89233123

[20] Ifuku S, Nogi M, Abe K, Handa K, Nakatsubo F and Yano H 2007 Surface modification of bacterial cellulose nanofibers for property enhancement of optically transparent composites: dependence on acetyl-group DS Biomacromolecules 8 1973-8.

[21] Cunha A, Zhou Q, Larsson P, Berglund L 2104 Topochemical acetylation of cellulose nanopaper structures for biocomposites: mechanisms for reduced water vapour sorption Cellulose 21 2773-2787.

[22] Agustin M B. Nakatsubo F and Yano H 2016 The thermal stability of nanocellulose and its acetates with different degree of polymerization Cellulose 23 451-64

[23] Okahisa Y, Yoshida A, Miyaguchi S and Yano H 2009 Optically transparent woodcellulose nanocomposite as a base substrate for flexible organic light-emitting diode displays Compos. Sci. Technol. 69 1958-61

[24] Yano H, Sugiyama, J, Nakagaito A N, Nogi M, Matsuura T, Hikita M and Handa K 2005 Optically transparent composites reinforced with networks of bacterial nanofibers. Adv. Mater. 17 153-5. 
[25] Nogi M, Handa K, Nakagaito A N and Yano H 2005 Optically transparent bionanofiber composites with low sensitivity to refractive index of the polymer matrix, Appl. Phys. Lett. 87243110

[26] Hsieh M C, Koga H, Suganuma K and Nogi M 2016 Hazy transparent cellulose nanopaper Sci. Rep. [Manuscript Under Peer Review]

[27] Iwamoto S, Abe K and Yano H 2008 The effect of hemicelluloses on wood pulp nanofibrillation and nanofiber network characteristics Biomacromolecules 9 1022-6

[28] Osch T H J, Perelaer J, Laat A W M and Schubert U S 2008 Inkjet printing of narrow conductive tracks on untreated polymeric substrates Adv. Mater. 20 343-5

[29] Kim C, Nogi M, Suganuma K and Yamato Y 2012 Inkjet-printed lines with well-defined morphologies and low electrical resistance on repellent pore-structured polyimide films ACS Appl. Mater. Interfaces 4 2168-73

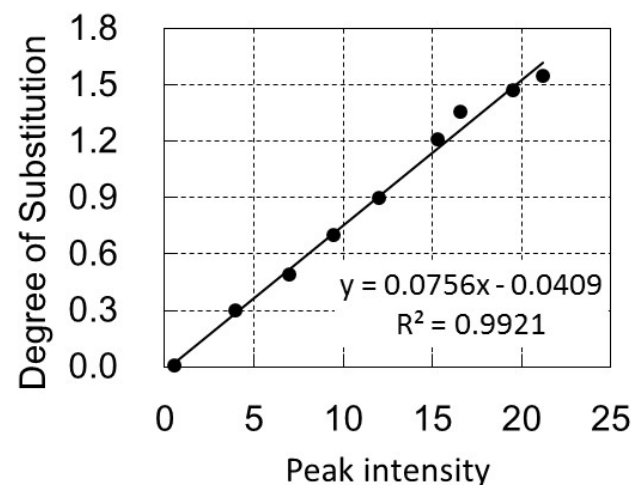

Figure 1. Calibration curve to determine the degree of substitution from the FTIR peak intensity.



$\begin{array}{llllllllllllllllll}a & b & c & d & e & f & g\end{array}$

Figure 2. Hazes of cellulose nanopaper from holocellulose pulps (a) and acetylated holocellulose pulps (b). Hazes of acetylated cellulose nanopaper rinsed with water (c), ethanol (d), methanol (e), isopropanol (f), or toluene (g). 

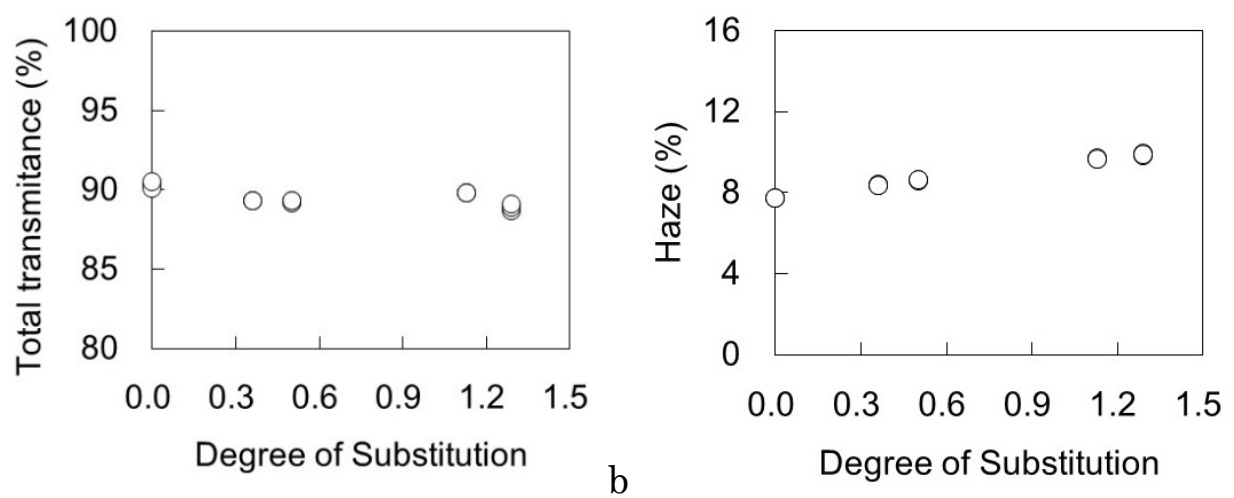

Figure 3. Total transmittance (a) and haze (b) of acetylated cellulose nanopaper for various degrees of substitution.

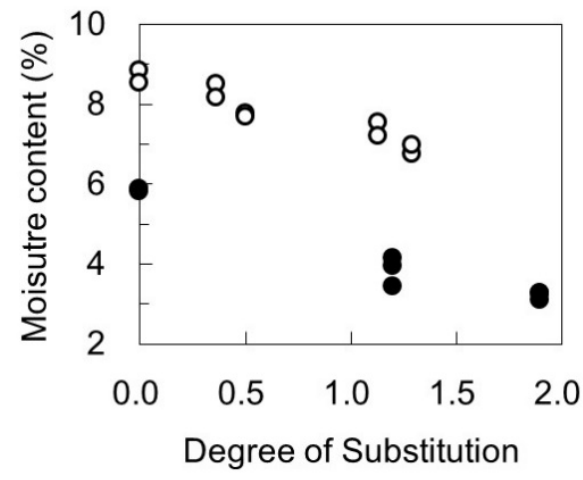

Figure 4. Moisture contents of acetylated cellulose nanopaper from holocellulose pulps (filled circles) and hardwood bleached kraft pulps (unfilled circles) under 55\% relative humidity and $20^{\circ} \mathrm{C}$.

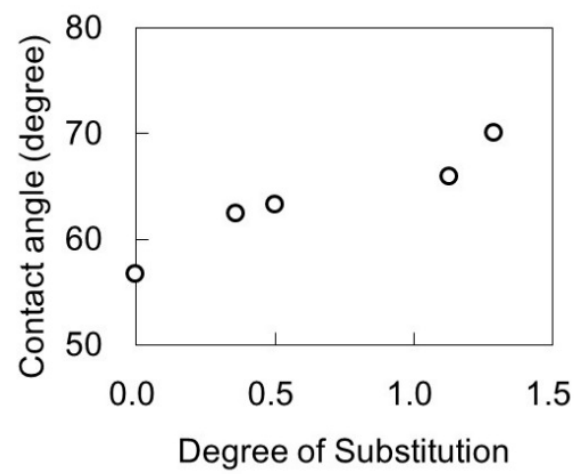

Figure 5. Contact angles of acetylated cellulose nanopaper for various degrees of substitution. 


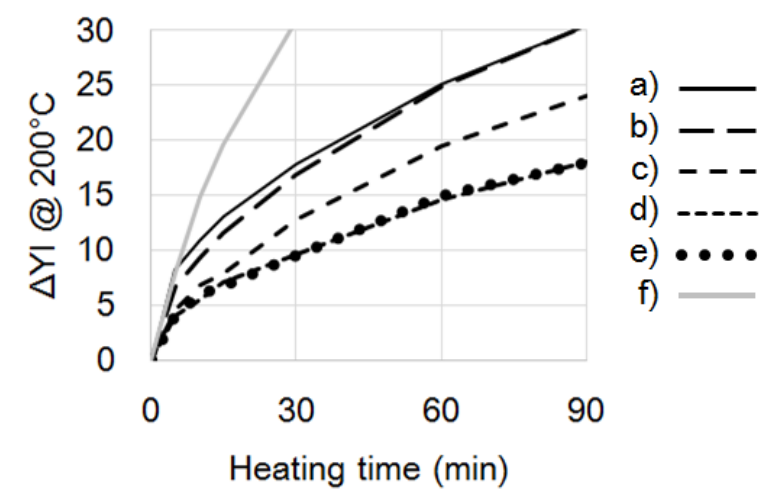

Figure 6. Differential yellowness index $(\Delta \mathrm{YI})$ curves of nanopaper at $200{ }^{\circ} \mathrm{C}$ in air. Transparent cellulose nanopaper with $\mathrm{DS}=0$ (a), DS=0.36 (b), DS=0.5 (c), DS=1.13 (d), DS=1.29 (e), and cellophane film (f).

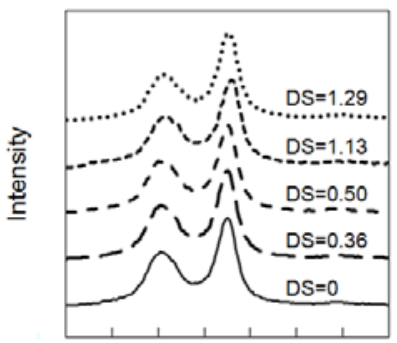

$\begin{array}{llllllll}5 & 10 & 15 & 20 & 25 & 30 & 35 & 40\end{array}$ a)

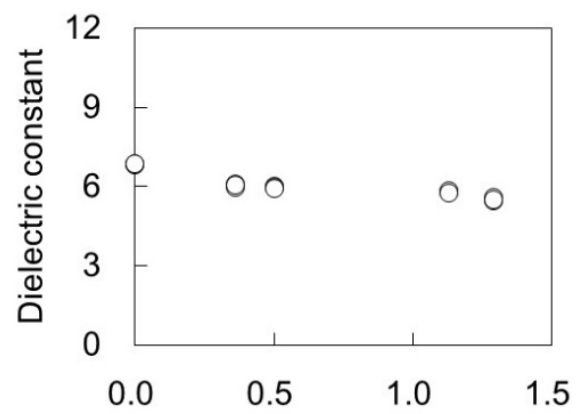

c) Degree of Substitution
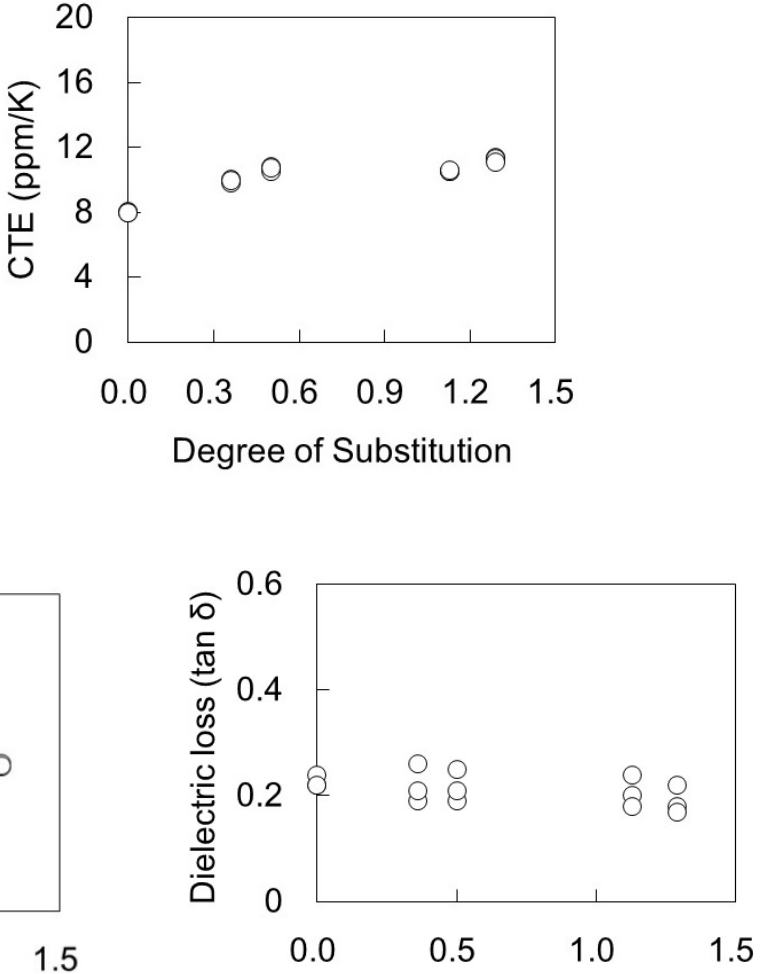

d)

Figure 7. Physical properties of acetylated transparent cellulose nanopaper for various degrees of substitution. X-ray diffraction profiles (a), coefficients of thermal expansion (CTE) (b), dielectric constant (c), and dielectric loss (tan $\delta)(d)$.

\section{Supporting information}

Table S1. Optical, hygroscopic, thermal, and other properties of transparent films. 


\begin{tabular}{|c|c|c|c|c|c|c|}
\hline & $\begin{array}{l}\text { Haze } \\
(\%)\end{array}$ & $\begin{array}{l}\text { Moisture } \\
\text { content } \\
(\%)\end{array}$ & $\begin{array}{l}\text { Contact } \\
\text { angle } \\
\left({ }^{\circ}\right)\end{array}$ & $\begin{array}{l}\text { coefficients } \\
\text { of thermal } \\
\text { expansion } \\
(\mathrm{ppm} / \mathrm{K})\end{array}$ & $\begin{array}{l}\text { Dielectric } \\
\text { constant }\end{array}$ & $\begin{array}{l}\Delta \mathrm{YI}=15 \\
\text { at } 200{ }^{\circ} \mathrm{C} \\
(\min )\end{array}$ \\
\hline $\begin{array}{r}\text { Acetylated } \\
\text { nanopaper } \\
(\mathrm{DS}=1.29)\end{array}$ & 9.8 & 6.5 & 70.1 & 10.5 & 5.8 & 65 \\
\hline $\begin{array}{l}\text { Native } \\
\text { nanopaper } \\
(\mathrm{DS}=0)\end{array}$ & 7.8 & 9.2 & 56.7 & 8.0 & 6.9 & 20 \\
\hline Cellophane ${ }^{* 1}$ & 2.7 & 9.0 & 43.5 & $\begin{array}{l}18.2^{* 2} \\
33.6{ }^{* 3}\end{array}$ & 8.0 & 10 \\
\hline $\mathrm{PET}^{*}$ & 7.3 & 0.4 & 74.6 & 26.6 & 3.1 & $>120$ \\
\hline Polyimide *5 & 4.0 & 0.6 & 74.3 & 24.6 & 3.4 & $>120$ \\
\hline
\end{tabular}

*1 : PT\#300, Rengo Co., Ltd

$*_{2}:$ in the flow direction

$* 3:$ in the transverse direction

*4 : Teijin Tetoron Film G2, Teijin DuPont Films Japan Limited

*5 : Kapton 300H, Du Pont-Toray Co., Ltd 